\title{
Inhibition of SIRT1 Transcription in Resveratrol-differentiated
}

\section{Medulloblastoma Cells}

\author{
Jing-Xin Ma, Hong Li, Xiao-Xin Cheng, Mo-Li Wu, Li-Jun Yu, Qing-You Kong, \\ Ming Liu, Jia Liu
}

Liaoning Laboratory of Cancer Genetics and Epigenetics and Department of Cell Biology, Dalian Medical University, Dalian 116044, China

Corresponding author: Professor Jia Liu, Liaoning Laboratory of Cancer Genetics \& Epigenetics and Department of Cell Biology, Dalian Medical University, Dalian 116044, China.

Submission date: April 24, 2013; Acceptance date: May 31, 2013; Publication date: May 31, 2013

\begin{abstract}
:
Backgrounds: Medulloblastoma (MB) is the commonest brain malignancy in childhood with poor prognosis, because of its rapid aggressive growth and frequent occurrence. The current chemotherapeutic regimens for medulloblastoma patients involve a combination of lomustine, cisplatin, carboplatin, vincristine or cyclophosphamide, which have distinct short- and long-term side-effects. It is therefore in urgent need to explore safer and more effective adjuvant approach(s). Resveratrol, a polyphenol rich in numerous plants, has multiple biological activities including anticancer effects. Our previous data confirmed that resveratrol inhibited proliferation and induced differentiation and apoptosis of medulloblastoma cells. SIRT1 is a deacetylase of class III HDACs and the supposed molecular effecter of resveratrol. SIRT1 involves in aging prevention and cancer formation in a cell-context specific manner. Nevertheless, the datum concerning the role(s) of SIRT1 in formation and prognosis of medulloblastoma is still missing.
\end{abstract}

Objective: The present study aimed to address the expression patterna of SIRT1 in medulloblastoma tissues and non-cancerous counterparts and to explore whether resveratrol exerts its anti-medulloblastoma effects via regulating SIRT1 expression and bioactivity.

Methods: The expression of SIRT1 in medulloblastoma and non-cancerous counterparts was elucidated by immunohistochemical ataining (IHC). To clarify the function of SIRT1 in medulloblastomas, SIRT1 expression in UW228-3 medulloblastoma cells were suppressed by 
RNA interference (RNAi). The influence of resveratrol in SIRT1 expressions in UW228-3 cells was analyzed by reverse transcription-polymerase chain reaction (RT-PCR), immunocytochemistry (ICC) and Western blotting (WB). The catalytic activity of deacetylase SIRT1 was examined by measuring the acetylation of the main substrate p53.

Results: IHC staining revealed that SIRT1 was expressed in $64.17 \%$ of MB tissues, which was higher than that in noncancerous cerebellum tissues (14.29\%). The frequencies of SIRT1 expression in the nodular MB $(22.22 \%)$ with better prognosis is lower than that in anaplastic MB (79.07\% ) and classic MB (60.29\%; P<0.05). The proliferation of UW228-3 cells was remarkably suppressed after being transfected with SIRT1 siRNA, accompanied with extensive cell death. The results of RT-PCR and WB showed that after 48 hours $100 \square \mathrm{M}$ resveratrol treatment, SIRT1 expression in UW228-3 cells was down-regulated at both transcriptional and translational levels. However, resveratrol has no effect on the deacetylase activity of SIRT1.

Conclusion: The above findings suggested that SIRT1 expression is corrected with the formation and prognosis of human MB. Resveratrol influences SIRT1 functioning in human MB cells through inhibiting SIRT1 expression rather than modulating its acetylation activity.

Keywords: resveratrol, SIRT1, RNA interference, deacetylase, medulloblastoma

\section{BACKGROUND:}

Medulloblastoma (MB) is the commonest brain tumor in childhood. Due to rapid growth, high invasiveness and frequent recurrence, the prognosis of MB is very poor. Although the therapy for MBs has been improved and the disease-free survival rates have been more or less increased, MB is still incurable for about 1/3 patients [1]. Moreover, prognosis is worse for patients less than 3 years old, due to inadequate degree of resection. As an important part of therapy, chemotherapy can reduce risk of recurrence, which is usually fatal. It is noteworthiness that drugs widely used such as lomustine, cisplatin, cyclophosphamide etc, bring severe unwanted side-effects and cause long-term disability. Therefore, it is impending to find safer and more effective drugs or potentiators for clinical management of MBs.

\section{INTRODUCTION:}

Resveratrol (Res), a polyphenol rich in various plants, has multiple beneficial activities, such as extending lifespan and delaying the onset of diseases associated with aging. Since 1997 [2], the cancer preventive effects of Res have been extensively demonstrated [3-7]. It is, therefore, considered as an excellent candidate for potentiator of platinum treatment [8]. Difficulties of drug delivery across the blood-brain barrier and failure to eliminate cancer stem cells are believed to be the major causes of recurrences in children with MB [9]. It is worthy noted that Res can be transported across blood-brain barrier through simple diffusion [10]. Our group 
demonstrated that Res could significantly inhibit the proliferation and induced differentiation and apoptosis of MB cells [11]. For this reason, we tried to gain insights into the mechanism(s) underlying the anti-medulloblastoma effects of resveratrol.

SIRT1, the most studied member of Sirtuins family, has been demonstrated to play roles in various biological processes, such as cell growth, differentiation and apoptosis. There is considerable debate on the roles of SIRT1 in tumorigenesis. In some cancers, SIRT1 is a tumor promoter [12-14], on the contrary, in other cancers, it acts as a tumor suppressor [15-17]. So far, it is widely accepted that SIRT1 functions in a cell-tissue context specific manner. It has been reported that SIRT1 involves in the differentiation of neural progenitors [18-19] and involves in the formation of neural tumor [20]. Limited studies in MB showed that inhibition of SIRT1 expression can induce the apoptosis of DAOY and MED283 MB cells [21]. However, in vivo data about roles of SIRT1 in MBs is absent. Our current study aimed to address this issue.

\section{MATERIALS AND METHODS:}

\section{Tissue collection and tissue microarray-based immunohistochemical staining}

With the consent of patients, MB tissues blocks were collected and prepared in tissue microarrays as described previously [22]. Immunohistochemical staining/IHC was performed on the sections from microarray. Briefly, deparaffinizing and rehydrating the sections, then, retrieving the antigen. After blocked with normal serum, the sections were incubated with solution containing primary polyclonal SIRT1 antibody (1:200, Santa Cruz Biotechnology, Inc, USA). Then the sections were incubated with a horseradish peroxidase conjugated secondary antibody, followed by development with diaminobenzidine and counter-staining with hematoxylin. Experienced pathology technicians reviewed and scored 10 separate fields for each slide. IHC staining was graded on a scale (Table.1) [23].

Table.1 IHC Scale for SIRT1 in different cerebellum tissues

\begin{tabular}{cc}
\hline Scale & Positive staining in cells \\
\hline- & no \\
+ & $<20 \%$ \\
++ & $21 \sim 50 \%$ \\
+++ & $>50 \%$ \\
\hline
\end{tabular}

Cell culture and treatment: Human UW228-3 MB cells were incubated in DMEM medium supplemented with $10 \%$ fetal bovine serum and cultured in a incubator with $5 \% \mathrm{CO}_{2}$ at $37 \mathrm{C}$. The cells were sub-cultured and treated with siRNA sequences against SIRT1 (SIRT1 siRNA) and 100uMRes, respectively. Three independent SIRT1 siRNA and controls were designed 
and synthesized by Shanghai GenePharma Co., Ltd, China. Res were purchased from Sigma-Aldrich.Co and resolved with DMSO and DMEM medium, respectively, to a stock concentration at $100 \mathrm{mM}$.

Evaluation of SIRT1 expression: In order to examine the influences of SIRT1 siRNA and Res on MB cells, RT-PCR, ICC and WB were conducted to measure the expression of SIRT1 at transcriptional and translational levels. In RT-PCR, the primer sequences for Sirt1 are: 5'-TCAGTGTCATGGTTCCTTTGC-3' (upward) and 5'-AATCTGCTCCTTTG CCACTCT-3' (downward) (620bp). The reaction were performed according the condition in relate article [24]. The primer sequences for control $\beta$-actin are 5'-GCATGGAGTCCTGTGGCAT-3' (upward) and 5'-CTAGAAGCATTTGCGG TGG-3' (downward) (326bp) [25]. In ICC, the polycolonal anti human SIRT1 antiboby was diluted at 1:200 and then added on the coverslips beared with MB cells. WB analyses were performed according previous article [25]. Briefly, prepare lysate from cell culture and determine the protein concentration. Load equal amounts of protein into the wells of the SDS-PAGE gel, along with the molecular weight markers. Then transfer the protein from the gel to the nitrocellulose membrane. After blocking in the 5\% blocking buffer in TBS, the membranes were staining with appropriate dilutions of primary antibody SIRT1 (1:800) and p53(acetyl K382) (1:500). Finally, incubate the membrane with solution containing secondary antibody and acquire image using techniques for chemiluminesence.

Flow cytometry (FCM) analysis: To determine the effects of SIRT1 silencing on cell proliferation, UW228-3 cells treated with or without SIRT1 siRNA were analyzed by FCM. The cells were detached with $0.25 \%$ trypsin, washed three times with phosphate buffered saline (PBS; pH7.4) and then fixed with 75\% ethanol. The cell samples were re-suspended in $0.5 \mathrm{ml}$ PBS and stained with propidium iodide (PI) in the darkness for 30 minutes. The DNA contents were measured by fluorescence-activated cell sorting/FACS on a Becton-Dickinson FACScan flow cytometry system and the data were analyzed using a Cellfit software (Becton, Dickinson and Company).

\section{Statistic analysis}

The discrepancy of SIRT1 expression in MB tissue and non-cancerous lesions were evaluated by non-parametric Kruskal-Wallis test (SPSS 11.5).

\section{RESULTS:}

\section{Up-regulated SIRT1 expression in MB tissues}

The expression of SIRT1 protein were detected by IHC staining in 4 tissue microarrays containing $120 \mathrm{MBs}$ and 7 tumor-surrounding noncancerous tissues. As shown in Table 2, the overall frequency of SIRT1 expression in cancerous tissues is $64.17 \%$ (77/120), which is higher than that $(14.29 \% ; 1 / 7)$ in noncancerous cerebellar tissues $(\mathrm{P}=0.009)$. 
Table 2. Expression of SIRT1 in different cerebellum lesions

\begin{tabular}{|c|c|c|c|c|c|}
\hline \multicolumn{2}{|c|}{ Histology } & \multirow[t]{2}{*}{ Positive } & \multicolumn{2}{|c|}{ - } & \multirow[t]{2}{*}{+} \\
\hline & & & $\geq++$ & & \\
\hline Noncancerous & & $1 / 7(14.29)$ & 6 & 1 & 0 \\
\hline \multirow{5}{*}{ medulloblastoma cell } & Large & $34 / 43$ & 0 & & \\
\hline & & $(79.07 \%)^{* *}$ & 9 & 19 & 15 \\
\hline & Classic & $41 / 68$ & 27 & 19 & 22 \\
\hline & & $(60.29 \%)^{* *}$ & 21 & 17 & \\
\hline & Nodular & $2 / 9(22.22 \%)$ & 7 & 1 & 1 \\
\hline
\end{tabular}

* statistical significance $(\mathrm{p}<0.05)$ compared with noncancerous tissues.

** statistical significance $(\mathrm{p}<0.05)$ compared with nodular medulloblastoma.

\section{Differential SIRT1 expression in three medulloblastoma subtypes}

MB tissues were classified into three morphological groups: large cell/anaplastic MB ( $n=43)$, classic MB ( $n=68)$ and nodular/demoplastic MB ( $n=9)$. Analysis of IHC data revealed that the positive rates of SIRT1 protein were different in the three subtypes (Table 2). Statistical analysis revealed that the frequencies of SIRT1 expression in the anaplastic/large cell (79.07\%) and the classic MB (60.29\%) have no statistical difference (P>0.05), but they are higher than that of the nodular/demoplastic (22.22\%) subtypes $(\mathrm{P}<0.05)$. According to clinical reports [26-27], the patients with the nodular MB usually have better prognosis than the other two subtypes.

\section{SIRT1-specific RNAi caused growth inhibition and apoptosis}

To explore the role of SIRT1 in survival of MB cells and validate the conclusion of IHC, the viability was detected in MB cells treated with SIRT1 siRNA and mock siRNA. As first, the expression of SIRT1 was manipulated by introducing three SIRT1-siRNA candidates into UW228-3 cells, respectively (Table 3).

Table 3 SIRT1 siRNA sequences $\left(5^{\prime} \rightarrow 3^{\prime}\right)$

\begin{tabular}{ccc}
\hline & \multicolumn{1}{c}{ sense } & \multicolumn{1}{c}{ antisense } \\
2 & GGAUGAAAGUGAAAUUGAA & UUCAAUUUCACUUUCAUCC \\
3 & AGCUGUUGGUCAAGACUAA & UUAGUCUUGACCAACAGCU \\
Negative control & UUCAUGAUGUUUGUGUGCUA & UAGCACACAAACAUCAUGC \\
Positive control (p53) & CUACUUCCUGAAAAACAACG & CGUUGUUUUCAGGAAGUAG \\
\hline
\end{tabular}

The inhibitory influences were evaluated by RT-PCR and WB. The results revealed that the SIRT1 siRNA sequence-1 exhibited better inhibitory effects (about 80\%) on SIRT1 transcription and translation, in contrast, no SIRT1 down-regulation was observed in cells transfected with mock siRNA [Figure 1A]. The effects of SIRT1 siRNA on proliferation and 
apoptosis were estimated in the cells treated with or without SIRT1 siRNA sequence-1 for 48 hours. H\&E staining and FCM assay revealed that the growth of the cells was suppressed and the apoptosis fractions and proportion of cell at G1 phage increased (Figure 1B).
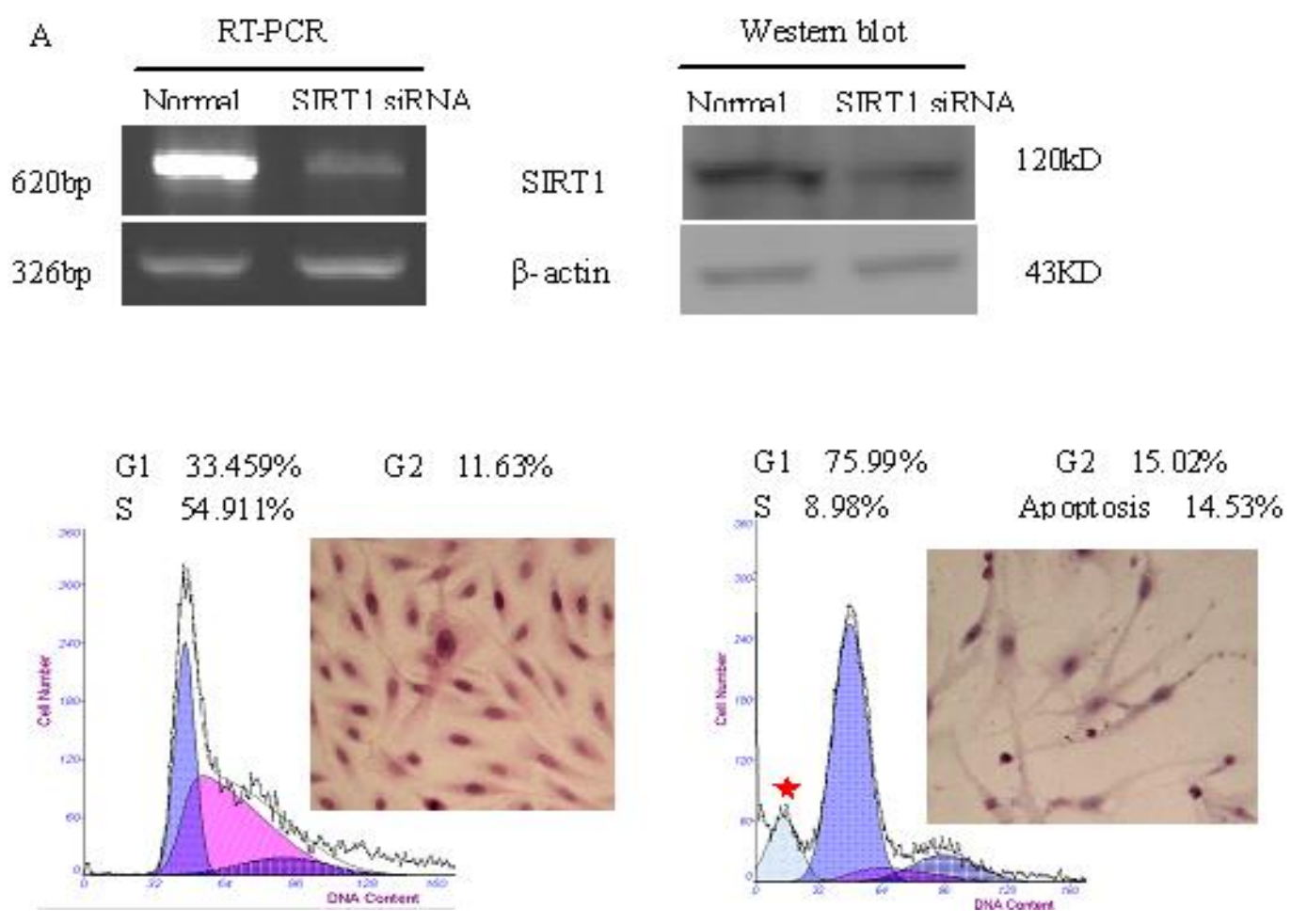

Figure 1. Selective silencing of SIRT1 by specific siRNA transfection and its biological effects on UW228-3 cells.

A. RT-PCR and Western-blot evaluation of the effects of SIRT1 siRNA on SIRT1 expression at transcriptional and translational levels, which were both downregulated. B. H\&E staining showed the decrease of cell number after silencing SIRT1, which was further comfirmed by the FCM analysis. SIRT1 siRNA induced the G1 arrest and appearance of the peak of apoptosis.

Res suppressed expression but not activity of SIRT1 in MB cells: To clarify potential correlation of the anti-MB activity of Res with its effects on SIRT1 expression, SIRT1 expression in UW228-3 MB cells with and without Res treatment. The results of RT-PCR, ICC and Western blotting reveal that the expression of SIRT1 decreased at both transcriptional and translational level after Res treatment [Figure 2A, 2B]. As a deacetylase, SIRT1 can mediate lots of biological processes by deacetylating target proteins. SIRT1 can suppress the pro-apoptotic activity of $\mathrm{p} 53$ by removing the acetylated lysine residue and promote the survival of cancer cells [28-29]. Since Res is often referred as the activator of SIRT1 [30], the acetylating level of p53 at 382 lysine has been examined, which shows that the level of p53 (k 382) did not changed significantly after treated with 100uM Res for 48hrs. 

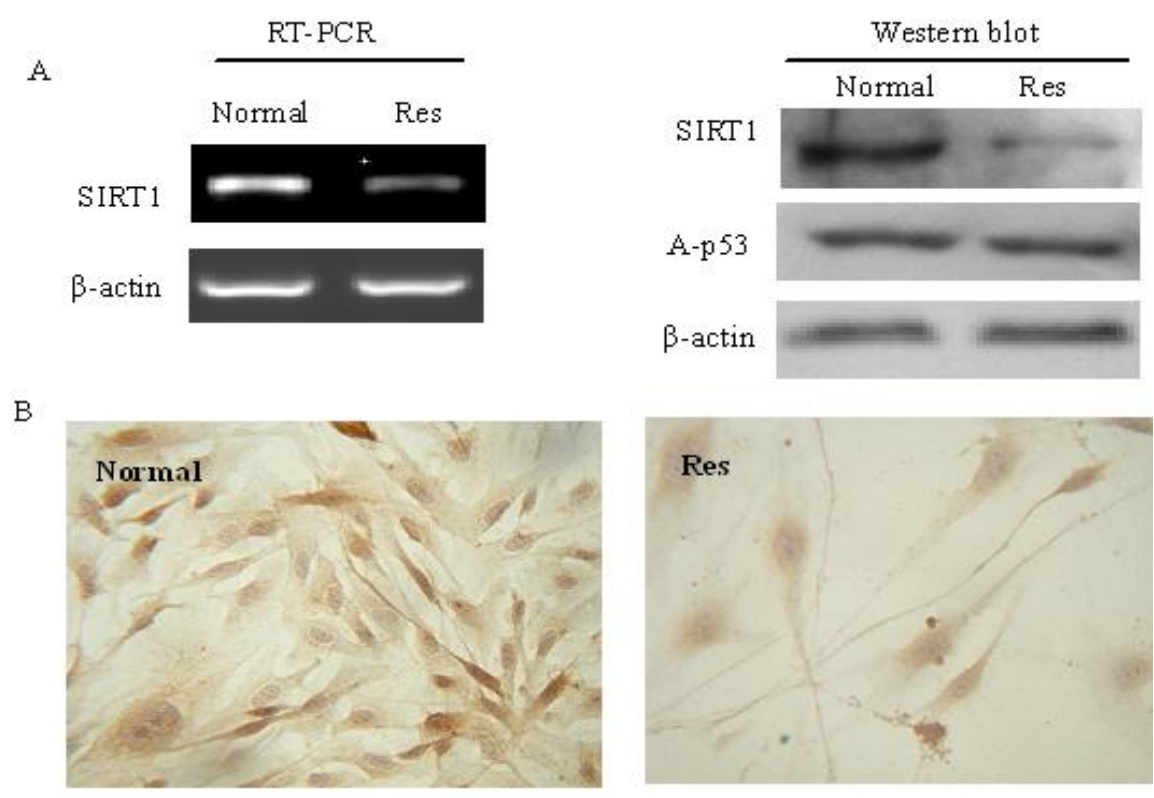

Figure 2. The effects of Res on the function of SIRT1 in UW228-3 cells.

A. The expression of SIRT1 at transcriptional and translation level were both decreased after treated with Res. But Res did not decrease the enzymatic activity of deacetylase SIRT1 as expected by measuring the acetylated p53, the main substrate of SIRT1. B. Similarly, ICC staining ascertained the diminished of SIRT1 protein.

\section{DISCUSSION:}

Sirtuins are found in all organisms and act as the critical regulators at the crossroads between cancer and aging [31,32]. SIRT1, a well understood member of Sirtuin family, modulates several cell signaling pathways in normal and malignant cells [33, 34]. It prevents cell aging and apoptosis of normal cells [35, 36], while the data obtained from human cancers are controversial because this protein is considered as a tumor promoter in neuroblastomas as well as prostate and skin cancers [37-39], but as a tumor suppressor in colon and breast cancers $[40,41]$. The conflicting data imply the cell type-related expression and functioning of SIRT1 in human malignancies [42]. It has been proven that SIRT1 is one of the major determinants of neural progenitor cell differentiation [43, 44]. MB is thought to originate from the primitive neuroectodermal cells. We speculated that SIRT1 might play certain role(s) in MB.

Our in vivo data demonstrated that the overall frequency of SIRT1 expression in MB were higher than that in tumor-surrounding tissues. Furthermore, in three major MB subtypes, with different histological features, aggressiveness and the grades of differentiation, SIRT1 proteins expressed differentially. The clinical data revealed that the classic MB and the large cell $\mathrm{MB}$ were less differentiated with rapid growth, resulting in unfavorable clinical outcome; in contrast, the nodular MB were surrounded by extensive fibrogenic tissues with pale nodules of differentiating neuroblasts; consequently, patients with this type usually have relatively better prognosis [26,27]. Our IHC staining demonstrated that SIRT1 expressed more highly in large cell $\mathrm{MB}$ and classic $\mathrm{MB}$ than that in nodular $\mathrm{MB}$, indicating the potential link of SIRT1 expression with the biological behavior and poor prognosis of MB. To 
confirm this notion, it is necessary to suppress SIRT1 expression by RNAi. Our data showed the growth of UW228-3 cells transfected with SIRT1 siRNA was significantly suppressed, accompanied with remarkable G1 phase arrest and apoptosis, consistent with those conducted on human DAOY and D283MED MB cells [21]. UW228-3 cells used in current study are from a classic MB. Since all of them are sensitive to anti-SIRT1 treatments, it would be possible that inhibition of SIRT1 expression may be applicable to different MB subtypes and SIRT1 might be a potential molecular target in the adjuvant management of MB.

Our previous data demonstrating anti-tumor activity of Res and present study regarding SIRT1 as a tumor promoter in MB promote us to explore the involvement of SIRT1 in roles of Res. At present, we showed Res can decrease the expression of SIRT1 in UW228-3 MB cells. Moreover, inhibiting the expression of SIRT1 by RNAi suppressed the cell proliferation, caused G1 arrest and trigged apoptotic cell death of MB cells. These above findings propose a possibility that Res play anti-MB roles by inhibiting expression of SIRT1. It is worthy of mentioning that Res is usually referred as the activator of SIRT1 [45], which deacetylate some tumor suppressor such as p53, FOXOs and Ku70 [46-48]. SIRT1-mediated deacetylation of p53(K382) decreases p53-mediated apoptosis, and then promotes the survival of cells. Therefore, we propose a question whether the anti-MB activity of Res is associated with its effect on the catalytic activity of SIRT1. In present study, we detected the acetylation of p53, the well-known substrate of SIRT1. ICC and WB data shown the acetylation of p53 at lysine 382 did not change significantly. Regarding other reports question Res as SIRT1 activator [49] and some function of Res is independent of SIRT1 [50]. We speculated that in MB cells, Res inhibits the proliferation and induces apoptosis in a SIRT1 activity-independent manner, but mainly by suppress the expression of SIRT1.

\section{CONCLUSION:}

Collectively, high SIRT1 expression might boost the formation of medulloblastoma. Resveratrol, a safe polyphenol and easy to intake from common foods, might possess anti-medulloblastoma by regulation the expression but not the enzymatic activity of SIRT1. Suppressing expression of SIRT1 might be a potential in therapy of medulloblastoma.

\section{REFERENCES:}

1. Kim YH, Cho SH, Lee SJ, Choi SA, Phi JH, Kim SK, Wang KC, Cho BK, Kim CY. Growth-inhibitory effect of neurotrophin-3-secreting adipose tissue-derived mesenchymal stem cells on the D283-MED human medulloblastoma cell line. J Neuro-oncol 2012; 106:89-98.

2. Jang M, Cai L, Udeani GO, Slowing KV, Thomas CF, Beecher CW, Fong HH, Farnsworth NR, Kinghorn AD, Mehta RG, Moon RC, Pezzuto JM. Cancer chemopreventive activity of resveratrol, a natural product derived from grapes. Science 1997; 275:218-20.

3. Baur JA, Sinclair DA. Therapeutic potential of resveratrol: the in vivo evidence. Nat Rev Drug Discov 2006; 5:493-506. 
4. Li ZG, Hong T, Shimada Y, Komoto I, Kawabe A, Ding Y, Kaganoi J, Hashimoto Y, Imamura M. Suppression of N-nitrosomethylbenzylamine (NMBA)-induced esophageal tumorigenesis in F344 rats by resveratrol. Carcinogenesis 2002; 23:1531-6.

5. Athar M, Back JH, Tang X, Kim KH, Kopelovich L, Bickers DR, Kim AL. Resveratrol: a review of preclinical studies for human cancer prevention. Toxicol. Appl. Pharmacol 2007; 224:274-83.

6. Niles RM, Cook CP, Meadows GG, Fu YM, McLaughlin JL, Rankin GO. Resveratrol is rapidly metabolized in athymic (nu/nu) mice and does not inhibit human melanoma xenograft tumor growth. J. Nutr 2006; 136:2542-6.

7. Kimura Y, Okuda H. Resveratrol isolated from Polygonum cuspidatum root prevents tumor growth and metastasis to lung and tumor-induced neovascularization in Lewis lung carcinoma-bearing mice. J. Nutr 2001; 131:1844-9.

8. Björklund M, Roos J, Gogvadze V, Shoshan M. Resveratrol induces SIRT1- and energy-stress-independent inhibition of tumor cell regrowth after low-dose platinum treatment. Cancer Chemother Pharmacol 2011; 68:1459-67.

9. Yu L, Baxter PA, Zhao X, Liu Z, Wadhwa L, Zhang Y, Su JM, Tan X, Yang J, Adesina A, Perlaky L, Hurwitz M, Idamakanti N, Police SR, Hallenbeck PL, Blaney SM, Chintagumpala M, Hurwitz RL, Li XN. A single intravenous injection of oncolytic picornavirus SVV-001 eliminates medulloblastomas in primary tumor-based orthotopic xenograft mouse models. Neuro Oncol 2011; 13:14-27.

10. Wang Q, Xu J, Rottinghaus GE, Simonyi A, Lubahn D, Sun GY, Sun AY. Resveratrol protects against global cerebral ischemic injury in gerbils. Brain Res 2002; 958:439-47.

11. Wang Q, Li H, Wang XW, Wu DC, Chen XY, Liu J. Resveratrol promotes differentiation and induces Fas-independent apoptosis of human medulloblastoma cells. Neurosci Lett 2003; 351:83-6.

12. Huffman DM, Grizzle WE, Bamman MM, Kim JS, Eltoum IA, Elgavish A, Nagy TR. SIRT1 is significantly elevated in mouse and human prostate cancer. Cancer Res 2007; 67:6612-8.

13. Hida Y, Kubo Y, Murao K, Arase S. Strong expression of a longevity-related protein, SIRT1, in Bowen's disease. Arch Dermatol Res 2007;299:103-6.

14. Bradbury CA, Khanim FL, Hayden R, Bunce CM, White DA, Drayson MT, Craddock C, Turner BM. Histone deacetylases in acute myeloid leukaemia show a distinctive pattern of expression that changes selectively in response to deacetylase inhibitors. Leukemia 2005; 19:1751-9.

15. Wang RH, Zheng Y, Kim HS, Xu X, Cao L, Luhasen T, Lee MH, Xiao C, Vassilopoulos A, Chen W, Gardner K, Man YG, Hung MC, Finkel T, Deng CX. Interplay among BRCA1, SIRT1, and Survivin during BRCA1-associated tumorigenesis. Mol Cell 2008; 32:11-20.

16. Firestein R, Blander G, Michan S, Oberdoerffer P, Ogino S, Campbell J, Bhimavarapu 
A, Luikenhuis S, de Cabo R, Fuchs C, Hahn WC, Guarente LP, Sinclair DA. The SIRT1 deacetylase suppresses intestinal tumorigenesis and colon cancer growth. PLoS ONE 2008; 3:e2020.

17. Wang RH, Sengupta K, Li C, Kim HS, Cao L, Xiao C, Kim S, Xu X, Zheng Y, Chilton B, Jia R, Zheng ZM, Appella E, Wang XW, Ried T, Deng CX. Impaired DNA damage response, genome instability, and tumorigenesis in SIRT1 mutant mice. Cancer Cell 2008; 14:312-23.

18. Zhang Y, Wang J, Chen G, Fan D, Deng M. Inhibition of Sirt1 promotes neural progenitors toward motoneuron differentiation from human embryonic stem cells. Biochem Biophys Res Commun 2011; 404:610-4.

19. Ichi S, Boshnjaku V, Shen YW, Mania-Farnell B, Ahlgren S, Sapru S, Mansukhani N, McLone DG, Tomita T, Mayanil CS. Role of Pax3 acetylation in the regulation of Hes1 and Neurog2. Mol Biol Cell 2011;22:503-12.

20. Qu Y, Zhang J, Wu S, Li B, Liu S, Cheng J. SIRT1 promotes proliferation and inhibits apoptosis of human malignant glioma cell lines. Neurosci Lett 2012; 525:168-72.

21. Baxter EW, Milner J. p53 Regulates LIF expression in human medulloblastoma cells. J Neuro-oncol 2010; 97:373-82.

22. Sun Y, Chen XY, Liu J et al. Differentiation caspase-3 expression in oncancerous, premalignant and cancer tissues of stomach and its clinical implication. Cancer Detect. Prevent 2006; 30: 168-17.

23. Petersen RP, Campa MJ, Sperlazza $J$ et al. Tumor infiltrating Foxp3+ regulatory T-cells are associated with recurrence in pathologic stage I NSCLC patients. Cancer 2006; 107: 2866-2872.

24. Ford J, Jiang M, Milner J. Cancer-specific functions of SIRT1 enables human epithelial cancer cell growth and survival. Cancer Res 2005; 65: 10457-1046.

25. Ma JX, Zhang KL, Liu X et al. Concurrent expression of aryl hydrocarbon receptor and CYP1A1 but not CYP1A1 MspI polymorphism is correlated withgastric cancers raised in Dalian, China. Cancer Lett 2006; 240:253-260.

26. Lamont JM, McManamy CS, Pearson AD, Clifford SC, Ellison DW. Combined histopathological and molecular cytogenetic stratification of medulloblastoma patients. Clin Cancer Res 2004; 10: 5482-5493.

27. Rossi A, Caracciolo V, Russo G, Reiss K, Giordano A. Medulloblastoma: from molecular pathology to therapy. Clin Cancer Res 2008; 14:971-976.

28. Li L, Wang L, Li L, Wang Z, Ho Y, McDonald T, Holyoake TL, Chen W, Bhatia R. Activation of p53 by SIRT1 inhibition enhances elimination of CML leukemia stem cells in combination with imatinib. Cancer Cell 2012; 21:266-81.

29. Howitz KT, Bitterman KJ, Cohen HY, Lamming DW, Lavu S, Wood JG, Zipkin RE, Chung P, Kisielewski A, Zhang LL, Scherer B, Sinclair DA. Small molecule activators of sirtuins extend Saccharomyces cerevisiae lifespan. Nature 2003; 425:191-6.

30. Borra MT, Smith BC, Denu JM. Mechanism of human SIRT1 activation by 
resveratrol. J Biol Chem 2005; 280:17187-17195.

31. Yamamoto H, Schoonjans K, Auwerx J. Sirtuin functions in health and disease. Mol Endocrinol 2007; 21:1745-1755

32. Lim CS. SIRT1: cellular senescence, cancer and organismal aging? Med Hypotheses 2006; 67: 989-990.

33. Jung-Hynes B, Ahmad N. Role of p53 in the antiproliferative effects of Sirt1 inhibition in prostate cancer cells. Cell Cycle 2009; 8:1478-1483.

34. Kim MJ, Ahn K, Park SH et al. SIRT1 regulates tyrosine hydroxylase expression and differentiation of neuroblastoma cells via FOXO3a. FEBS Lett 2009; 583:1183-1188

35. Law IK, Liu L, Xu A et al. Identification and characterization of proteins interacting with SIRT1 and SIRT3: implications in the anti-aging and metabolic effects of sirtuins. Proteomics 2009; 9: 2444-2456

36. Kim D, Nguyen MD, Dobbin MM et al. SIRT1 deacetylase protects against neurodegeneration in models for Alzheimer's disease and amyotrophic lateral sclerosis. EMBO J 2007; 26: 169-3179

37. Yu W, Fu YC, Zhou XH et al. Effects of resveratrol on $\mathrm{H}(2) \mathrm{O}(2)$-induced apoptosis and expression of SIRTs in H9c2 cells. J Cell Biochem 2009; 107: 741-74711

38. Chu F, Chou PM, Zheng X et al. Control of multidrug resistance gene mdr1 and cancer resistance to chemotherapy by the longevity gene sirt1. Cancer Res 2005; 65: 10183-10187

39. Hida Y, Kubo Y, Murao K, Arase S. Strong expression of a longevity-related protein, SIRT1, in Bowen's disease. Arch Dermatol Res 2007; 299: 103-106

40. Firestein R, Blander G, Michan S et al. The SIRT1 deacetylase suppresses intestinal tumorigenesis and colon cancer growth. PLoS ONE 2008; 3: e202014

41. Wang RH, Zheng Y, Kim HS et al. Interplay among BRCA1, SIRT1, and Survivin during BRCA1-associated tumorigenesis. Mol Cell 2008; 32: 11-20 12

42. Fang Y, Nicholl MB. Sirtuin 1 in malignant transformation: friend or foe? Cancer Lett 2011; 306: 10-145

43. Ota H, Tokunaga E, Chang K et al. Sirt1 inhibitor, Sirtinol, induces senescence-like growth arrest with attenuated Ras-MAPK signaling in human cancer cells. Oncogene 2006; 25: 176-185

44. Kim D, Nguyen MD, Dobbin MM et al. SIRT1 deacetylase protects against neurodegeneration in models for Alzheimer's disease and amyotrophic lateral sclerosis. EMBO J 2007; 26: 3169-3179

45. Substrate-specific activation of sirtuins by resveratrol. Kaeberlein M, McDonagh T, Heltweg B, Hixon J, Westman EA, Caldwell SD, Napper A, Curtis R, DiStefano PS, Fields S, Bedalov A, Kennedy BK. J Biol Chem. 2005 Apr 29;280(17):17038-45.

46. Luo J, Nikolaev AY, Imai S, Chen D, Su F, Shiloh A, et al. Negative control of p53 by Sir2alpha promotes cell survival under stress. Cell 2001; 107:137-48

47. Brunet A, Sweeney LB, Sturgill JF, Chua KF, Greer PL, Lin Y, et al. Stress-dependent regulation of FOXO transcription factors by the SIRT1 deacetylase. Science 2004; 
303:2011-5.

48. Cohen HY, Miller C, Bitterman KJ, Wall NR, Hekking B, Kessler B, et al. Calorie restriction promotes mammalian cell survival by inducing the SIRT1 deacetylase. Science 2004; 305:390-2.

49. Beher D, Wu J, Cumine S, Kim KW, Lu SC, Atangan L, Wang M. Resveratrol is not a direct activator of SIRT1 enzyme activity. Chem Biol Drug Des 2009; 74: 619-24.

50. Wang Z, Li W, Meng X, Jia B. Resveratrol induces gastric cancer cell apoptosis via reactive oxygen species, but independent of sirtuin1. Clin Exp Pharmacol Physiol 2012; 39:227-32. 